Neuroepidemiology 2008;31:262-263

DOI: $\underline{10.1159 / 000165365}$

\section{Experiences from the Mumbai Stroke Register}

Thomas Truelsen

Department of Neurology, Rigshospitalet, Copenhagen University Hospital, Copenhagen, Denmark

The results in this issue of Neuroepidemiology from The Mumbai Stroke Register (MSR) are an important contribution increasing our knowledge about the stroke burden in an Indian urban population [1].

The standardized stroke incidence rate was 152/100,000 and comparable to those reported from populations in high-income countries (HICs), and the 28-day case fatality was high with a fatal outcome in 1 out of 3 stroke events. Complete case ascertainment of both hospitalized and non-hospitalized stroke events was ensured by ongoing contact to 120 local medical practitioners, and additionally, to local hospitals, nursing homes, diagnostic centres, and municipal health authorities. Data on first-ever stroke were collected during a 2-year period in a well-defined population with 337,391 permanent residents of which 156,000 were 25 years of age or older allowing estimations of incidence rates and case fatality. The researchers used the protocol from the World Health Organization stepwise approach to stroke surveillance (STEPS Stroke) [2] and the study is an example of how international collaboration can support building local capacity for the collection of community-based stroke data, which in turn may be used to urge local health authorities to focus on prevention of non-communicable diseases, including stroke.

In the MSR, the mean age of stroke patients was 66 years, 10 years lower than reported from stroke studies in HICs [3]. The low mean age of stroke patients suggests that the social consequences of stroke may be marked as patients may be core contributors to maintaining the income of the family, and survival with disability may reduce work opportunities and necessitate increased care by family members. One third of the Indian population lives below the poverty line defined as a monthly income of less than 5.5 EUR (rural) and 8.3 EUR (urban) [4]. Less than $20 \%$ of total health expenditure in India is public [5] and access to basic health care and medical treatment is very limited for a substantial part of the general population due to shortage of health professionals and for economic reasons. Continuous medical control of major stroke risk factors such as hypertension and diabetes is equally limited to the wealthier parts of the population [6].

The results from the MSR are supported by the results from another Indian community-based stroke study in Kolkata which reported an age-standardized stroke incidence rate of 145/100,000 while the 30 -day case fatality was $41 \%$ [7]. An important question from these two recent stroke incidence studies is whether stroke rates are currently changing or stable. In the MSR, history of hypertension was reported in $83 \%$ of stroke events and a recent study suggests that more than $54 \%$ of stroke events are attributable to high blood pressure and that over $80 \%$ of the attributable burden are found in low- and middle-income countries (LMICs) [8]. The MSR does not provide data on other major stroke risk factors but other studies have shown that smoking and diabetes are prevalent in large parts of the Indian population [9]. Stroke rates may therefore be on the rise, and with the low income in the general population public health interventions reducing the exposure to major stroke risk factors should be the chosen strategy to tackle the burden of stroke. Ongoing stroke surveillance in populations will remain outmost important for monitoring trends in stroke incidence and impact of interventions. Hopefully the Mumbai study is repeated within few years.

The MSR participated in the STEPS Stroke feasibility study [10] in which a shared protocol was used in 9 different study sites in 5 different countries. In the STEPS Stroke feasibility study data on hospitalized stroke patients were included (step 1), but the protocol was specifically developed with the aim of facilitating expansion of data collection to also include non-hospitalized events in the population, and to include reliable denominators for estimating rates. This was successfully done in the MSR and shows the potential for the stepwise approach to stroke surveillance where more complex data are registered as capacity for data collection develops locally. Adherence to the same protocol will increase comparability of results between sites and over time. The authors have recently published their experience using the STEPS Stroke protocol in their population which may serve as an inspiration for other sites [11].

Community-based stroke registries are essential for our understanding of trends in stroke occurrence, the burden of stroke in the population, and for monitoring the effect of interventions. To date, the majority of stroke registries are from populations in HICs with a long tradition in stroke epidemiology and in which many already had experienced declining stroke mortality rates [3]. Results have helped targeting areas for continuous improvements in prevention and better care. In contrast, little is known about stroke occurrence in LMICs, but the progress in data collection during the last few years from sites in India and other LMICs is promising and opens the potential for increased awareness of the stroke burden and for improved prevention.

\section{References}

1 Dalal PM, Malik S, Bhattacharjee M, Trivedi ND, Vairale J, Bhat P, Deshmukh S, Khandelwal K, Mathur VD: Population-based stroke survey in Mumbai, India: incidence and 28-day case fatality. Neuroepidemiology 2008;31:254-261.

2 World Health Organization's stepwise approach to stroke surveillance. www.who.int/chp/steps/stroke

\section{KARGER}

Fax +41613061234 E-Mail karger@karger.ch www.karger.com
(C) 2008 S. Karger AG, Base

$0251-5350 / 08 / 0314-0262 \$ 24.50 / 0$ 
3 Feigin VL, Lawes CM, Bennett DA, Anderson CS: Stroke epidemiology: a review of population-based studies of incidence, prevalence, and case-fatality in the late 20th century. Lancet Neurol 2003;2:43-53.

4 http://www.planningcommission.gov.in/news/prmar07.pdf

5 Musgrove P, Zeramdini R, Carrin G: Basic patterns in national health expenditure. Bull World Health Organ 2002;80:134-142.

6 Pandian JD, Srikanth V, Read SJ, Amanda GT: Poverty and stroke in India. Stroke 2007;38:3063-3069.

7 Das SK, Banerjee TK, Biswas A, Roy T, Raut DK, Mukherjee CS, Chaudhuri A, Hazra A, Roy J: A prospective community-based study of stroke in Kolkata, India. Stroke 2007;38:906-910.

8 Lawes CM, Vander Hoorn S, Rodgers A: Global burden of blood-pressure-related disease. Lancet 2001;371:1513-1518.

-9 Gupta R, Joshi P, Mohan V, Reddy KS, Yusuf S: Epidemiology and causation of coronary heart disease and stroke in India. Heart 2008;94: $16-26$.
10 Truelsen T, Heuschmann PU, Bonita R, Arjundas G, Dalal P, Damasceno A, Nagaraja D, Ogunniyi A, Oveisgharan S, Radhakrishnan K, Skvortsoya VI, Stakhovskaya V: Standard method for developing stroke registers in low-income and middle-income countries: experiences from a feasibility study of a stepwise approach to stroke surveillance (STEPS Stroke). Lancet Neurol 2007;6:134-139.

11 Dalal P, Bhattacharjee M, Vairale J, Bhat P: Mumbai Stroke Registry (2005-2006) - Surveillance using WHO steps stroke instrument: challenges and opportunities. J Assoc Physicians India 2008;56:675-680.

Thomas Truelsen, MD, PhD, DMSc

Department of Neurology, Rigshospitalet

Copenhagen University Hospital

DK-2200 Copenhagen (Denmark)

E-Mail truelsen@post3.tele.dk 